\title{
Naissance jumelée
}

Deutsch erschienen

in Nr. 32-33/2005
Dès 1996, la FMH a publié 12 thèses sur le sauvetage en Suisse, dans l'espoir d'unifier et d'améliorer le fonctionnement de la chaîne de sauvetage. Les auteurs avaient déjà formulé l'exigence, dans le cadre du forum de sauvetage de la FMH, selon laquelle les patients en détresse puissent être transférés directement, sous surveillance et traitement médical compétents, à l'hôpital destiné aux cas les plus graves. Dans ce but, la $10^{\mathrm{e}}$ des thèses susmentionnées demandait la création d'une grille unifiée pour la répartition en catégories de tous les hôpitaux en Suisse disposant d'un service d'urgence.

Conscient de l'acuité des effets que peuvent avoir une telle catégorisation, en termes politiques également, le forum de sauvetage a développé une grille permettant aux hôpitaux de sélectionner et de documenter eux-mêmes la catégorie à laquelle ils appartiennent parmi les quatre à disposition. Cette catégorisation est destinée aux médecins de premier recours et aux services de sauvetage, pour leur indiquer quels sont les hôpitaux qui admettent des blessés gravement et très gravement atteints et combien. Selon le forum de sauvetage, il n'y a guère de risque de voir des établissements se surestimer, toute situation sérieuse débordant immédiatement les limites d'un service d'urgence surévalué.

Depuis, les directeurs médicaux des services d'urgence se sont organisés en communauté d'intérêts (CI). Un premier projet a été d'établir un document par un groupe de travail sur les questions d'organisation et de structure des services d'urgence, revu de fond en comble après une large consultation en 2004. Ce document est destiné à servir de fil conducteur aux responsables dans l'agencement de leurs services. Pour des raisons didactiques, ces recommandations ont abouti à une répartition en trois groupes, selon la fonction du service dans son environnement (grandeur et importance de l'hôpital, lits par région, nombre de patients, etc.), et non selon des critères d'affectation. Cette communauté d'intérêts sera intégrée au cours de l'année au sein de la Société suisse de médecine d'urgence et de sauvetage (SSMUS) et exercera son activité en tant que sous-groupe de médecine d'urgence clinique. Les médecins de ce dernier secteur attachent une importance particulière à une bonne coopération avec les soins d'urgence.

Les deux documents - mis sur pied séparément - sont fortuitement dès lors à disposition en même temps. Etant donné que leurs objectifs sont différents et sans lien direct entre eux, leurs auteurs ont renoncé à les faire fusionner arbitrairement et à les présenter sous cette forme au public.

L'expérience et les suggestions des utilisateurs diront si nos objectifs sont atteints, à savoir simplifier et raccourcir la voie vers la prise en charge définitive des patients.

Dr M. Müller, Dr K. Meier

\section{Catégories de services d'urgence*}

Forum de sauvetage de la FMH

\section{Introduction}

Des exigences ou des directives manuscrites et contraignantes existent dans presque tous les domaines spécialisés. Jusqu'ici, le domaine très important de la médecine d'urgence ne disposait pas de telles recommandations. C'est la raison pour laquelle le Forum de sauvetage de la FMH a tenté de combler cette lacune par le biais de la catégorisation suivante. Les hôpitaux décident eux-mêmes de la catégorie à laquelle ils veulent appartenir. Ceux qui se sont attribués une basse catégorie ont bien entendu tout loisir d'aménager leur service spécialisé de meilleure manière que ce qui est fixé dans les exigences. Il n'y a pas de catégorie apte à couvrir chaque cas et chaque situation. Ces catégories doivent constamment être revues et adaptées. Elles doivent également être transparentes pour le médecin référant. Elles ne sauraient néanmoins servir de base aux indemnités des assurances. 


\section{Services d'urgence} chirurgicale

E: exigé

S: souhaitable

$\mathrm{P}$ : possible

\begin{tabular}{|c|c|c|c|c|}
\hline & \multicolumn{4}{|c|}{ Catégories } \\
\hline & I & II & III & IV \\
\hline \multicolumn{5}{|l|}{ Organisation } \\
\hline Urgences - chirurgie/traumatologie & $\mathrm{E}$ & $\mathrm{E}$ & $\mathrm{E}$ & - \\
\hline Chirurgie/lignes directrices en traumatologie & $\mathrm{E}$ & $\mathrm{E}$ & $\mathrm{E}$ & $\mathrm{E}$ \\
\hline Equipe de traumatologie & $\mathrm{E}$ & $\mathrm{E}$ & $\mathrm{E}$ & $\mathrm{E}$ \\
\hline Responsables pour les directives et l'organisation & $\mathrm{E}$ & $\mathrm{E}$ & $\mathrm{E}$ & $\mathrm{E}$ \\
\hline \multicolumn{5}{|l|}{ Départements/services } \\
\hline Chirurgie & $\mathrm{E}$ & $\mathrm{E}$ & $\mathrm{E}$ & - \\
\hline Neurochirurgie & $\mathrm{E}$ & $\mathrm{E}$ & - & - \\
\hline Traumatologie/orthopédie & $\mathrm{E}$ & $\mathrm{E}$ & $\mathrm{E}$ & - \\
\hline Anesthésie & $\mathrm{E}$ & $\mathrm{E}$ & $\mathrm{E}$ & - \\
\hline Chirurgie pédiatrique & $\mathrm{E}$ & - & - & - \\
\hline \multicolumn{5}{|c|}{ Disponibilité clinique (spécialistes immédiatement à disposition 24 h sur 24) } \\
\hline \multicolumn{5}{|l|}{ Plan de service } \\
\hline Chirurgie & $\mathrm{E}$ & $\mathrm{E}$ & $\mathrm{E}$ & $\mathrm{S}$ \\
\hline Plan de supervision (assistants) & $\mathrm{E}$ & $\mathrm{E}$ & $S$ & - \\
\hline Anesthésie & $\mathrm{E}$ & $\mathrm{E}$ & $\mathrm{E}$ & $\mathrm{S}$ \\
\hline \multicolumn{5}{|l|}{ Garde et service $24 \mathrm{~h}$ sur 24} \\
\hline Chirurgie cardiaque & $\mathrm{E}$ & $\mathrm{S}$ & - & - \\
\hline Chirurgie de la main & $\mathrm{E}$ & $S$ & - & - \\
\hline Chirurgie plastique & $\mathrm{E}$ & $S$ & - & - \\
\hline Chirurgie viscérale/chirurgie générale & $E$ & $\mathrm{E}$ & $\mathrm{E}$ & - \\
\hline Neurochirurgie & $E$ & $E$ & $S$ & - \\
\hline Ophtalmologie & $\mathrm{E}$ & $\mathrm{E}$ & $S$ & - \\
\hline Chirurgie des maxillaires & $\mathrm{E}$ & $\mathrm{E}$ & $S$ & - \\
\hline Orthopédie/traumatologie & $\mathrm{E}$ & $\mathrm{E}$ & $\mathrm{E}$ & $S$ \\
\hline Soins intensifs & $\mathrm{E}$ & $\mathrm{E}$ & $S$ & - \\
\hline Radiologie & $\mathrm{E}$ & $\mathrm{E}$ & $\mathrm{E}$ & $\mathrm{S}$ \\
\hline Chirurgie thoracique & $\mathrm{E}$ & $\mathrm{E}$ & $S$ & - \\
\hline Chirurgie pédiatrique & $\mathrm{E}$ & $S$ & - & - \\
\hline \multicolumn{5}{|l|}{ Personnel du service d'urgence } \\
\hline Directeur du service d'urgence & E & $\mathrm{E}$ & $\mathrm{E}$ & $\mathrm{E}$ \\
\hline \multicolumn{5}{|l|}{ Service d'urgence/salle de réveil } \\
\hline Présence du personnel soignant 24 h sur 24 & $\mathrm{E}$ & $\mathrm{E}$ & $\mathrm{E}$ & - \\
\hline \multicolumn{5}{|l|}{ Soins intensifs } \\
\hline Service de soins intensifs & $\mathrm{E}$ & $E$ & $S$ & - \\
\hline \multicolumn{5}{|l|}{ Anesthésie } \\
\hline Présence 24 h sur 24 & $\mathrm{E}$ & $E$ & $S$ & - \\
\hline Sur appel 24 h sur 24 & - & - & $\mathrm{E}$ & $\mathrm{E}$ \\
\hline \multicolumn{5}{|l|}{ Services de radiologie (24 h sur 24 ) } \\
\hline Technicien diplômé en radiologie médicale & $E$ & $\mathrm{E}$ & $S$ & $S$ \\
\hline Sonographie & $\mathrm{E}$ & $E$ & $\mathrm{E}$ & $\mathrm{S}$ \\
\hline Tomographie informatisée & $\mathrm{E}$ & $E$ & $P$ & $P$ \\
\hline Angiographie & $\mathrm{E}$ & $\mathrm{E}$ & $S$ & - \\
\hline IRM & $P$ & $\mathrm{P}$ & - & - \\
\hline \multicolumn{5}{|l|}{ Laboratoire (24 h sur 24) } \\
\hline Sur place & $\mathrm{E}$ & $E$ & $S$ & $\mathrm{~S}$ \\
\hline Accord avec un laboratoire externe & - & - & $\mathrm{E}$ & $\mathrm{E}$ \\
\hline
\end{tabular}




\section{Services d'urgence non- chirurgicale (médecine)}

E: exigé

S: souhaitable

P: possible

\begin{tabular}{|c|c|c|c|c|}
\hline & \multicolumn{4}{|c|}{ Catégories } \\
\hline & I & II & III & IV \\
\hline \multicolumn{5}{|l|}{ Organisation } \\
\hline Urgence non chirurgicale ou cas d'urgence intégré & $\mathrm{E}$ & $\mathrm{E}$ & $\mathrm{E}$ & $\mathrm{E}$ \\
\hline Directives médicales & $\mathrm{E}$ & $\mathrm{E}$ & $\mathrm{E}$ & $\mathrm{E}$ \\
\hline Responsables pour les directives et l'organisation & $\mathrm{E}$ & $\mathrm{E}$ & $\mathrm{E}$ & $\mathrm{E}$ \\
\hline \multicolumn{5}{|l|}{ Départements/services } \\
\hline Médecine & $\mathrm{E}$ & $\mathrm{E}$ & $\mathrm{E}$ & - \\
\hline Anesthésie & $\mathrm{E}$ & $\mathrm{E}$ & $\mathrm{E}$ & - \\
\hline Pédiatrie & $\mathrm{E}$ & $\mathrm{E}$ & - & - \\
\hline \multicolumn{5}{|l|}{ Premier avis par le chef de clinique en médecine interne/assistants } \\
\hline Plan de service pour les internistes parmi les spécialistes & $\mathrm{E}$ & $\mathrm{E}$ & $\mathrm{E}$ & $\mathrm{E}$ \\
\hline \multicolumn{5}{|l|}{ Disponibilité clinique des spécialistes } \\
\hline \multicolumn{5}{|l|}{ Cardiologie } \\
\hline Auprès du patient 24 h sur 24 dans les & $10 \mathrm{~min}$. & $60-120$ & sur appel & - \\
\hline Angioplastie coronaire transluminale percutanée (ACTP) & $20 \mathrm{~min}$. & - & - & - \\
\hline \multicolumn{5}{|l|}{ Neurologie/Neuroradiologie } \\
\hline Auprès du patient 24 h sur 24 , dans les & $10 \mathrm{~min}$. & $60-120$ & - & - \\
\hline Intervention intra-artérielle & $30 \mathrm{~min}$. & - & - & - \\
\hline \multicolumn{5}{|l|}{ Gastroentérologie/endoscopie } \\
\hline Auprès du patient 24 h sur 24 , dans les & $10 \mathrm{~min}$. & $60-120$ & sur appel & - \\
\hline \multicolumn{5}{|l|}{ Pneumologie/endoscopie } \\
\hline Sur appel 24 h sur 24 , dans les & $10 \mathrm{~min}$. & $60-120$ & sur appel & - \\
\hline \multicolumn{5}{|c|}{ Néphrologie/rhumatologie/hématologie/oncologie/infectiologie } \\
\hline Sur appel 24 h sur 24 , dans les & $60 \mathrm{~min}$. & $60-120$ & sur appel & - \\
\hline \multicolumn{5}{|l|}{ Psychiatrie } \\
\hline Sur appel 24 h sur 24 , dans les & $30 \mathrm{~min}$. & 120 & sur appel & - \\
\hline \multicolumn{5}{|l|}{ Radio-oncologie } \\
\hline Sur appel 24 h sur 24 & $30 \mathrm{~min}$. & - & - & - \\
\hline \multicolumn{5}{|l|}{ Equipe de transplantation et suivi médical } \\
\hline Auprès du patient 24 h sur 24 & $30 \mathrm{~min}$. & - & - & - \\
\hline \multicolumn{5}{|l|}{ Pédiatrie } \\
\hline Sur appel 24 h sur 24 & $30 \mathrm{~min}$. & $60-120$ & sur appel & - \\
\hline \multicolumn{5}{|l|}{ Personnel du service d'urgence } \\
\hline Responsable du service d'urgence & $\mathrm{E}$ & $\mathrm{E}$ & $\mathrm{E}$ & $\mathrm{E}$ \\
\hline \multicolumn{5}{|l|}{ Salle de réveil } \\
\hline Présence du personnel soignant 24 h sur 24 & $\mathrm{E}$ & $\mathrm{E}$ & $\mathrm{E}$ & - \\
\hline \multicolumn{5}{|l|}{ Soins intensifs } \\
\hline Service des soins intensifs & $\mathrm{E}$ & $\mathrm{E}$ & $S$ & - \\
\hline Service des soins intensifs en pédiatrie & $\mathrm{E}$ & S & - & - \\
\hline \multicolumn{5}{|l|}{ Anesthésie } \\
\hline Présence 24 h sur 24 & $\mathrm{E}$ & $E$ & $S$ & $S$ \\
\hline Sur appel 24 h sur 24 & - & - & $\mathrm{E}$ & $\mathrm{E}$ \\
\hline \multicolumn{5}{|l|}{ Services radiologiques (24 h sur 24 ) } \\
\hline Sonographie & $\mathrm{E}$ & $\mathrm{E}$ & $\mathrm{E}$ & $S$ \\
\hline Tomographie informatisée & $\mathrm{E}$ & $\mathrm{E}$ & $\mathrm{P}$ & $P$ \\
\hline Angiographie & $\mathrm{E}$ & $\mathrm{E}$ & $S$ & - \\
\hline IRM & $P$ & $\mathrm{P}$ & - & - \\
\hline \multicolumn{5}{|l|}{ Laboratoire (24 h sur 24 ) } \\
\hline - Sur place & $\mathrm{E}$ & $\mathrm{E}$ & $\mathrm{S}$ & $S$ \\
\hline - Accord avec un laboratoire externe & - & - & $\mathrm{E}$ & $\mathrm{E}$ \\
\hline
\end{tabular}

